\title{
GEM operation in double-phase xenon
}

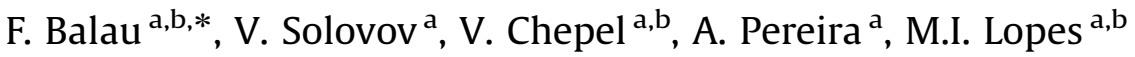 \\ a LIP-Coimbra, Department of Physics, University of Coimbra, Rua Larga, P-3004-516 Coimbra, Portugal \\ ${ }^{\mathrm{b}}$ CFRM, Department of Physics, University of Coimbra, Rua Larga, P-3004-516 Coimbra, Portugal
}

\section{A R T I C L E I N F O}

Available online 17 August 2008

\section{Keywords:}

Xenon

Double-phase detectors

Gas electron multiplier

GEM

Dark matter

\begin{abstract}
A B S T R A C T
Operation of Gas Electron Multiplier (GEM) in pure xenon was studied in a double-phase chamber under well-controlled conditions, in terms of temperature, pressure and gas purity. The maximum gain of $\approx 150$ was achieved in gas phase at $-108^{\circ} \mathrm{C}$. Stable operation, during several hours, was observed.
\end{abstract}

(c) 2008 Elsevier B.V. All rights reserved.

\section{Introduction}

Double-phase xenon (liquid/gas) detectors are becoming extensively used and continue to be developed by several groups and collaborations aiming at detection of galactic dark matter in the form of hypothetical heavy neutral particles with the mass of $\approx 10-\approx 1000 \mathrm{GeV}$ coupled to the ordinary matter through the weak interaction and generically called Weakly Interacting Massive Particles (WIMPs). WIMPs are expected to be observed through their elastic scattering from nuclei, of the detector material, thus resulting in nuclear recoils with the kinetic energy $\lesssim 100 \mathrm{keV}$ and approximately exponential spectrum. Extremely small predicted scattering cross-section leads to expected event rate of $\lesssim 1 \mathrm{event} / \mathrm{kg} /$ day, i.e. orders of magnitude lower than achievable natural radioactive gamma and electron background rate. Use of low background materials and active background discrimination techniques, as well as efficient shielding, are therefore mandatory for these experiments. Liquid xenon double-phase detectors, in which WIMP scattering in the liquid phase is followed by extraction of electrons from the recoil track to gas, offer both intrinsic amplification of the signal via secondary scintillation of gas and an efficient discrimination method by comparing the ratio of secondary to primary light [1-3]. The scintillation light is detected with photomultiplier tubes that are known to be a source of significant radioactive background. This justifies searches for alternative amplification techniques that would use only low radioactivity materials.

Micro-pattern avalanche detectors such as GEMs and alike seem to be good candidates for this purpose. They can be made

\footnotetext{
* Corresponding author at: LIP-Coimbra, Department of Physics, University of Coimbra, Rua Larga, P-3004-516 Coimbra, Portugal. Tel.: +351239410684; fax: +351239822358

E-mail address: balau@lipc.fis.uc.pt (F. Balau).
}

sensitive to the scintillation light by deposition of a thin layer of CsI photocathode on one of the surfaces [4]. However, operation of these devices in a two-phase system is not sufficiently studied. Promising results were obtained both with a triple GEM structure in double-phase argon, krypton and xenon [5], and with a single GEM placed above liquid xenon [6]. For xenon, a maximum gain of about 200 was reported for a triple GEM and about 25 with a single foil. Instabilities of GEM operation in saturated vapour sometimes leading to complete disappearance of the signal, presumably because of xenon condensation in the GEM channels, have also been noticed [7].

In this work we aimed at achieving a higher gain with single GEM by reducing the liquid temperature and thus the vapour density. From the experience with GEMs at room temperature, it is well known that the maximum achievable gain decreases with increasing of gas pressure [8]. Good temperature control and stability become more critical when approaching the xenon triple point. Therefore, special effort was made to ensure good temperature stabilisation of the experimental chamber. As a result, stable operation of GEM during several hours at the visible gain up to 150 has been achieved at the temperature of $-108^{\circ} \mathrm{C}$ using ${ }^{241} \mathrm{Am}$ as a source of $5 \mathrm{MeV} \alpha$-particles and $60 \mathrm{keV} \gamma$-rays.

\section{Experimental setup}

Measurements were carried out with the setup shown in Fig. 1(a).

A stainless steel cathode with ${ }^{241} \mathrm{Am} \alpha$-source mounted on top of it was placed at a distance of $11.1 \mathrm{~mm}$ below a $50 \mu \mathrm{m}$ thick GEM foil. The GEM, manufactured by $3 \mathrm{M}$ [9], has holes of $70 \mu \mathrm{m}$ in diameter at $140 \mu \mathrm{m}$ pitch. A collector grid made from $50 \mu \mathrm{m}$ stainless steel wires at $1 \mathrm{~mm}$ pitch was placed $3.5 \mathrm{~mm}$ above the GEM. The electrode structure was mounted on a stainless steel 
a

b

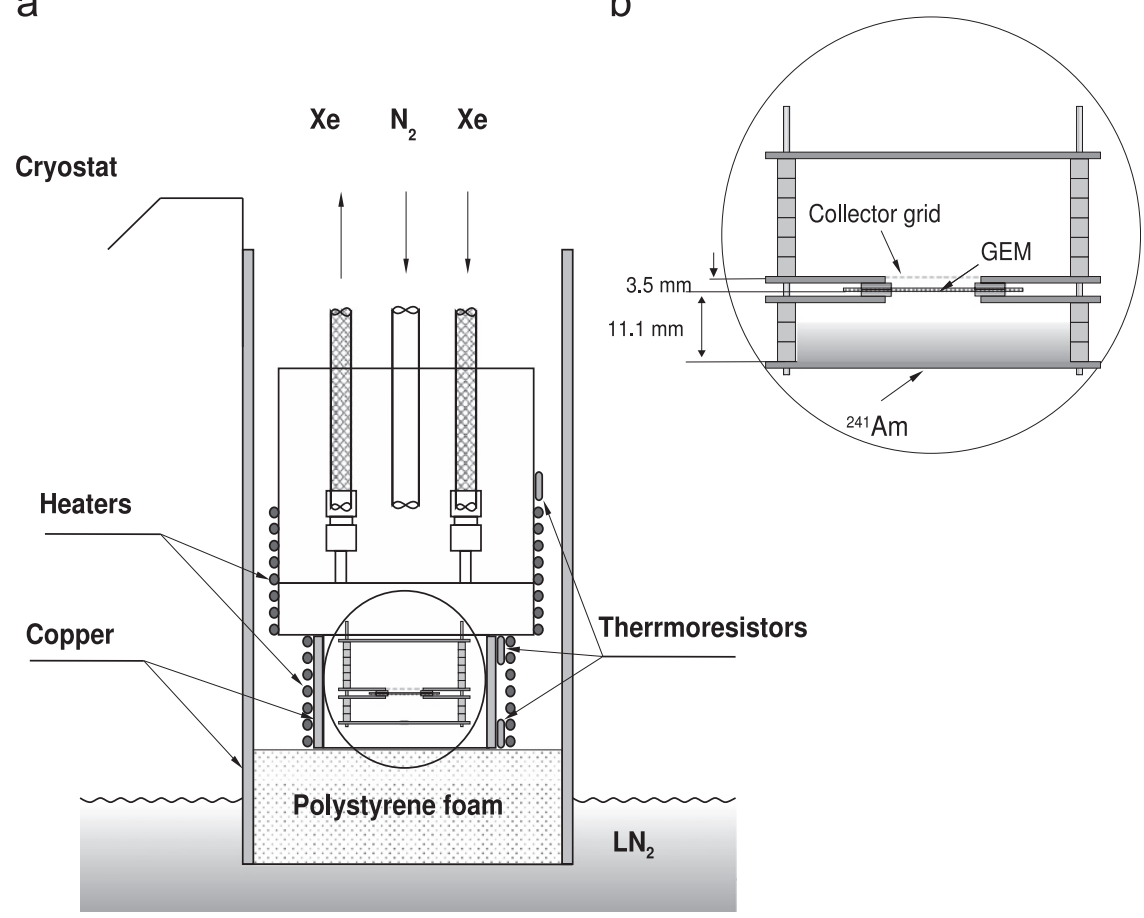

Fig. 1. Chamber in the cryostat.

flange with ceramic feedthroughs and placed into a cylindrical chamber, $100 \mathrm{~mm}$ diameter and $70 \mathrm{~mm}$ high, made of titanium. Inside the chamber, only high-vacuum compatible materials such as metal, glass, ceramic and PTFE were used. The chamber was placed inside of a liquid nitrogen cryostat as shown in Fig. 1(b). Special care was taken to ensure temperature stability and uniformity along the chamber. The copper screens around the chamber allowed to maintain a constant temperature gradient of about $2{ }^{\circ} \mathrm{C}$ between the bottom and the top of the chamber. The power delivered to the heaters was automatically adjusted according to the level of liquid nitrogen in the cryostat, which was measured with a capacitive probe, so that the temperature of the chamber could be maintained constant within $1{ }^{\circ} \mathrm{C}$. The temperature was measured with precision of $0.5^{\circ} \mathrm{C}$ using three platinum thermo-resistors distributed along the chamber. The chamber was helium leak tested, pumped to $10^{-7}$ mbar and baked at $75^{\circ} \mathrm{C}$ for three days. Then it was further cleaned by passage of xenon gas purified by Oxisorb column.

The chamber was filled with liquid xenon (purified with Oxisorb) up to a level of $7 \pm 1 \mathrm{~mm}$ above the $\alpha$-source. The voltage of $-4.5 \mathrm{kV}$ was applied to the cathode resulting in a field of $\approx$ $3 \mathrm{kV} / \mathrm{cm}$ in the liquid, at which about $50 \%$ of the electrons reaching the liquid-gas boundary are emitted into the gas phase [10]. It is worth mentioning that due to strong recombination, the charge that is extracted from the track of $\alpha$-particles in the liquid is only about $5 \%$ of that in the gas, i.e. about $3 \mathrm{fC}$ per $\alpha$-particle [11].

The readout schematics is presented in Fig. 2.

Low-noise charge-sensitive preamplifiers (Cremat-110) were connected to the bottom face of the GEM and to the collector grid. The output signals of the preamplifiers were amplified and shaped by a spectroscopy amplifier (Canberra 2021) and analysed by a multi-channel analyzer. Two modes of operation were used. In the first mode, the top face of the GEM and the collector grid were both kept at ground potential. In this case all the charge extracted from the liquid was collected at the bottom face

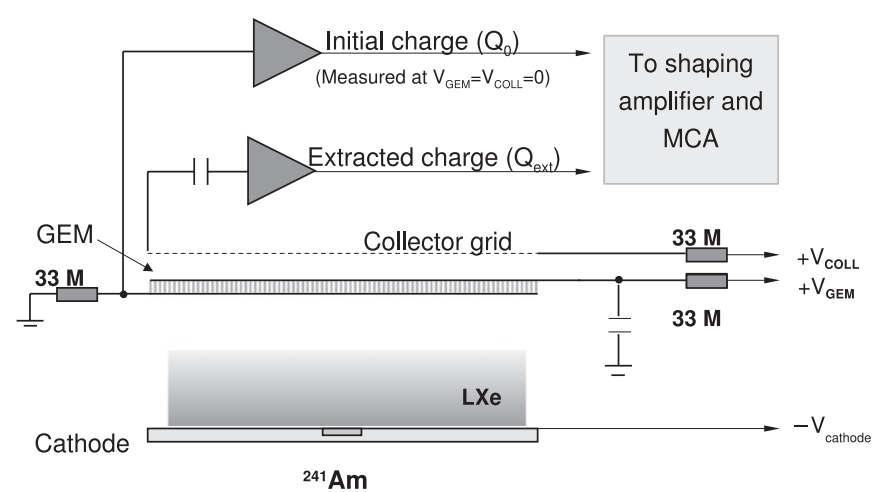

Fig. 2. Readout schematics.

of the GEM. This allowed to measure the initial charge $\left(Q_{0}\right)$ arriving to the GEM. In the second mode, the initial charge was amplified in the GEM and partially extracted to the collector grid $\left(Q_{\text {ext }}\right)$ by applying positive voltages to the GEM and collector grid. The amplification factor $Q_{\text {ext }} / Q_{0}$ (also referred herein as visible gain), was measured as a function of the voltage applied to the GEM $\left(V_{\mathrm{GEM}}\right)$ at constant voltage of $1.3 \mathrm{kV}$ applied to the collector grid.

\section{Results}

Amplitude spectra were recorded at different voltages (between 525 and $625 \mathrm{~V}$ ) across the GEM. Fig. 3 shows the spectra recorded for the highest visible gain $(\approx 150)$ achieved in this work.

In the upper spectrum, the main peak corresponds to $5.5 \mathrm{MeV}$ $\alpha$-particles from ${ }^{241} \mathrm{Am}$ and appears at lower energy that can be expected for the gamma rays of the same energy due to the strong 

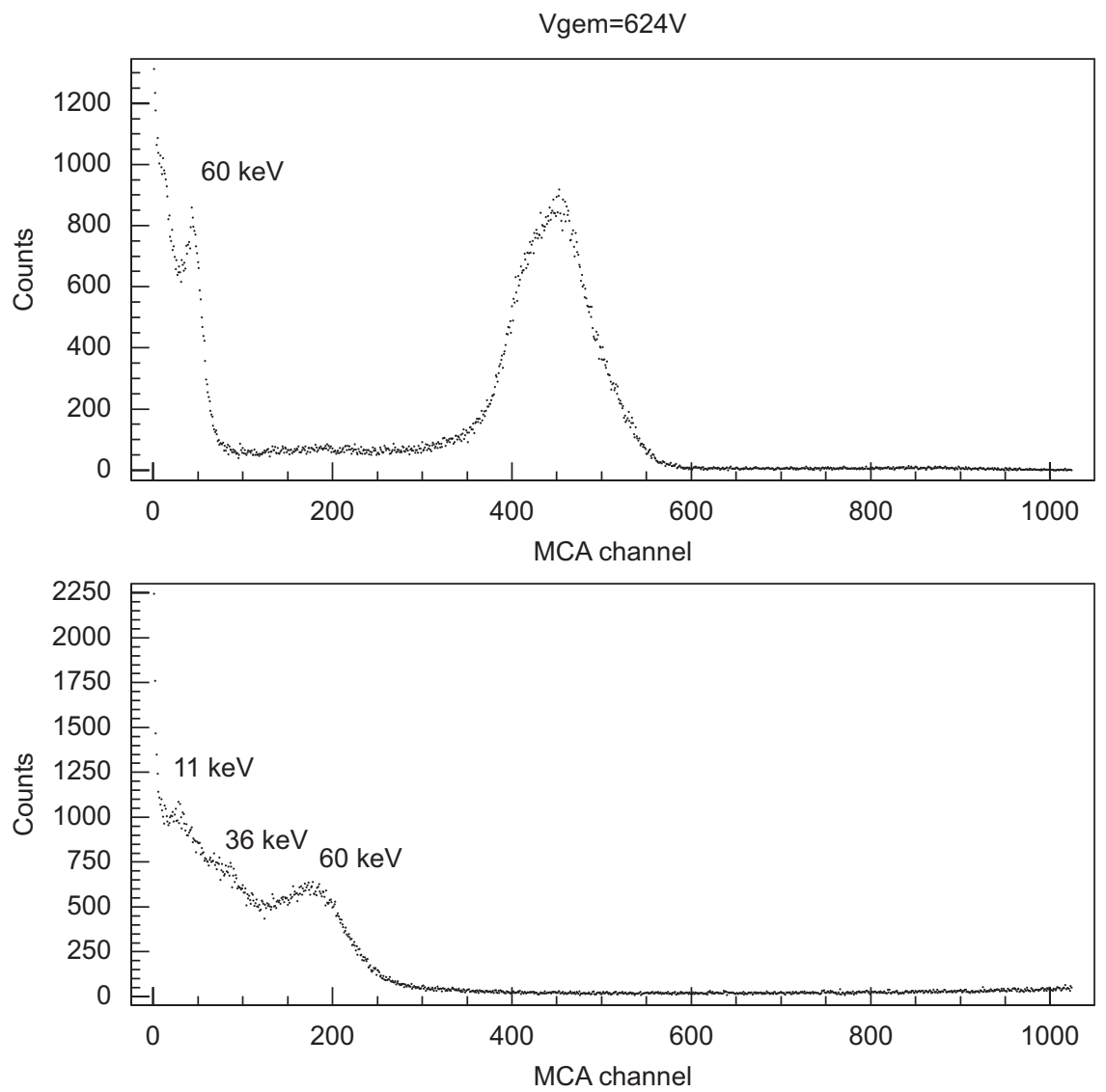

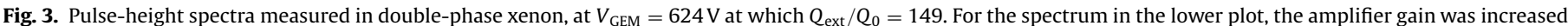
by a factor of 3 .

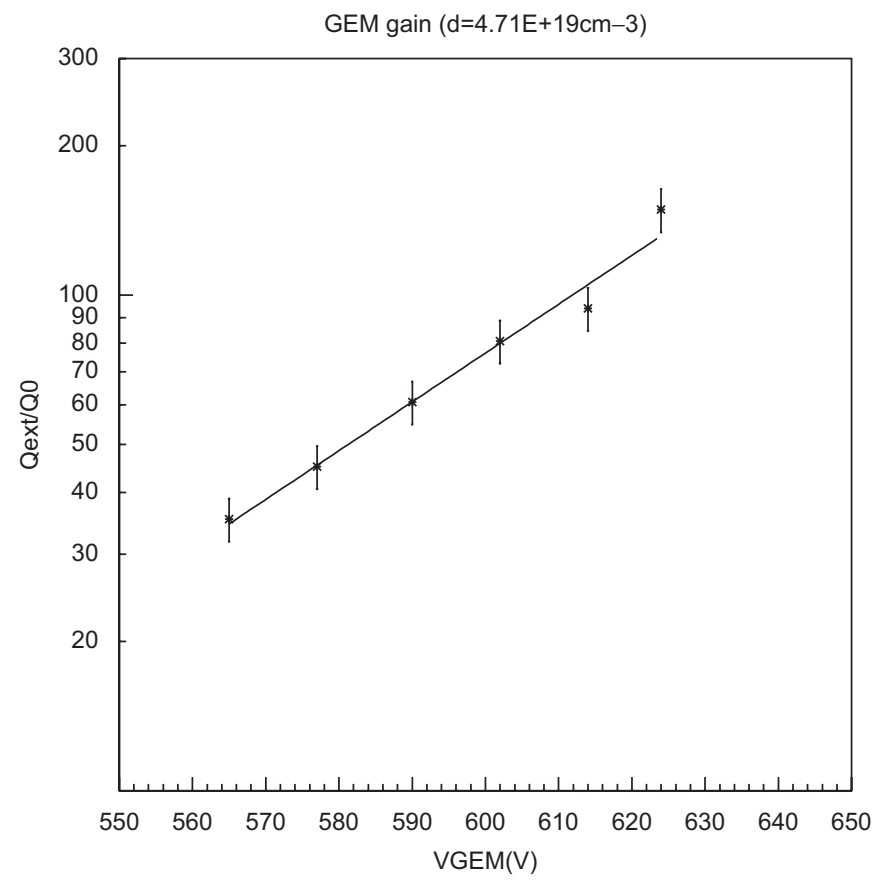

Fig. 4. Gain $\left(Q_{\text {ext }} / Q_{0}\right)$ as a function of the voltage applied across the GEM for $\left(V_{\text {coll }}=1.3 \mathrm{kV}\right)$.

recombination in LXe. The ${ }^{241} \mathrm{Am} 60 \mathrm{keV}$ gamma rays, as well as $\approx 30 \mathrm{keV}$ xenon escape peak are clearly visible in the spectra. Low energy ${ }^{237} \mathrm{~Np} \mathrm{X}$-rays can also be distinguished.
Table 1

Experimental conditions and maximum gain as measured in the present work and in Ref. [6]

\begin{tabular}{llllll}
\hline & $T\left({ }^{\circ} \mathrm{C}\right)$ & $P($ bar $)$ & Density $\left(\mathrm{cm}^{-3}\right)$ & $Q_{\text {ext }} / Q_{0}$ & $V_{\text {GEM }}(\mathrm{V})$ \\
\hline Ref. [6] & -102 & 1.40 & $6.10 \times 10^{19}$ & 25 & 650 \\
Present work & -108 & 1.04 & $4.71 \times 10^{19}$ & 150 & 624 \\
\hline
\end{tabular}

Fig. 4 shows the dependence of the visible gain on the voltage across the GEM. The GEM gain exponentially grows with $V_{\mathrm{GEM}}$, as expected.

The best energy resolution for $\alpha$-particles $(\approx 19 \%$, FWHM) was obtained at $602 \mathrm{~V}$ corresponding to the gain of 81 .

The maximum gain achieved in the present work and experimental conditions are compared with those of Ref. [6] in Table 1. A higher gain obtained in this work can be explained by the fact that the measurements were done at lower temperature, i.e. at lower gas density, being in general agreement with the observations at room temperature and high pressure [8].

\section{Conclusions}

Operation of single GEM in double-phase xenon was studied. Continuous stable operation, during several hours, was repeatedly observed.

The maximum gain of $\approx 150$ was achieved at $-108^{\circ} \mathrm{C}$ and vapour density of $4.71 \times 10^{19} \mathrm{~cm}^{-3}$. Energy resolution of $19 \%$ (FWHM) was obtained for $5.5 \mathrm{MeV} \alpha$-particles. 


\section{Acknowledgements}

This work was supported by Fundação para a Ciência e Tecnologia, Portugal (project POCI/FP/81928/2007).

\section{References}

[1] G.J. Alner, et al., Astropart. Phys. 28 (2007) 287.

[2] D.Yu. Akimov, et al., Astropart. Phys. 27 (2007) 46.
[3] E. Aprile, et al., New Astron. Rev. 49 (2005) 289.

[4] D. Mörmann, et al., Nucl. Instr. and Meth. A 471 (2001) 333.

[5] A. Bondar, et al., Nucl. Instr. and Meth. A 556 (2006) 273.

[6] V. Solovov, et al., Nucl. Instr. and Meth. A 580 (2007) 331.

[7] P.K. Lightfoot, private communication.

[8] A. Bondar, et al., Nucl. Instr. and Meth. A 419 (1998) 418

[9] P.S. Barbeau, et al., Nucl. Instr. and Meth. A 515 (2003) 439

[10] E.M. Gushchin, et al., Sov. Phys. J. Exp. Theor. Phys. 55 (1982) 650.

[11] E. Aprile, et al., Nucl. Instr. and Meth. A 307 (1991) 119. 